\title{
Somnolencia y calidad del sueño en estudiantes de medicina de una universidad peruana
}

\author{
Edmundo Rosales ${ }^{1}$, Martha Egoavil ${ }^{2}$, Claudia La Cruz ${ }^{3}$, Jorge Rey de Castro ${ }^{3,4}$
}

Resumen

Palabras clave
Objetivos: Determinar el grado de somnolencia diurna y calidad del sueño en estudiantes de medicina. Diseño: Estudio transversal. Lugar: Universidad Peruana Cayetano Heredia. Participantes: Estudiantes de medicina. Intervenciones: Muestra no probabilística de estudiantes de medicina del sexto(76 externos) y sétimo(74 internos) años de estudios. Cuestionarios: datos demográficos, escala de somnolencia de Epworth e índice de calidad del sueño Pittsburgh. Principales medidas de resultados: Somnolencia diurna y calidad del sueño. Resultados: No hubo diferencia con respecto a la somnolencia y calidad del sueño entre externos e internos. De todos los encuestados, 58\% tenía mala calidad del sueño y $34 \%$ excesiva somnolencia diurna. La latencia subjetiva del sueño mayor de 30 minutos, dormir 6 horas o menos, eficiencia subjetiva del sueño menor de 85\% y el uso de medicación hipnótica estuvieron asociados con mala calidad del sueño. Asimismo, dormir 5 horas o menos, consumo de cafeína y tabaquismo estuvieron asociados a mala calidad del sueño y excesiva somnolencia diurna. La correlación entre calidad del sueño y somnolencia diurna fue $R=0,426 p<0,001$. Conclusiones: Encontramos alta frecuencia de pobre calidad del sueño y excesiva somnolencia diurna en los estudiantes de medicina encuestados. Ambas estuvieron asociadas a consumo de café́na y tabaco, latencia subjetiva del sueño prolongada, corta duración del sueño, eficiencia subjetiva del sueño menor de 85\% y uso de medicación hipnótica. Hubo correlación entre calidad del sueño y somnolencia diurna.

Sueño; estudiantes de medicina; educación médica.
Sleepiness and sleep quality in medical students of a Peruvian university

\section{Abstract}

Objective: To determine the degree of excessive daytimesleepiness and sleep quality in medical students. Design: Cross-sectional survey. Setting: Universidad Peruana Cayetano Heredia. Participants: Medical students. Interventions: No probabilistic sample of the sixth (76 externs) and seventh (74 interns) year medical students. Questionnaires: demographic data, Epworth sleepiness scale and Pittsburgh sleep quality index. Main outcome measures: Daytime sleepiness and sleep quality. Results: There was no difference in scores obtained between both populations. Of the participants, $58 \%$ had poor sleep quality and $34 \%$ had excessive daytime sleepiness. To have a subjective sleep latency

\footnotetext{
1 Programa Maestría en Medicina. Facultad de Medicina, Universidad Peruana Cayetano Heredia. Lima, Perú.

2 Programa Maestría en Geriatría y Gerontología. Facultad de Medicina, Universidad Peruana Cayetano Heredia. Lima, Perú.

3 Universidad Peruana Cayetano Heredia. Lima, Perú.

4 Neumólogo y especialista en Trastornos Respiratorios del Sueño. Clínica Anglo Americana. Lima, Perú.
}

more than 30 minutes, to sleep 6 hours or less, sleep efficiency less than $85 \%$ and to use hypnotic medication were related with poor sleep quality. Also, to sleep 5 hours or less, to consume caffeine and tobacco were related to poor sleep quality and excessive daytime sleepiness. The correlation between sleep quality and daytime sleepiness was $R=0,426 p<0,001$. Conclusions: We found a high frequency of poor sleep quality and excessive daytime sleepiness in medical students. These parameters were associated to caffeine and tobacco consume, long subjective sleep latency, short sleep duration, sleep efficiency less than $85 \%$ and use of hypnotic medication. Also we found correlation between sleep quality and daytime sleepiness.

Key words: Sleep; students, medical; education, medical.

\section{INTRODUCCIÓN}

La duración del sueño requerida para que una persona joven se sienta despierta y alerta durante el día es en promedio 7 a 8 horas, en un período de 24 horas $\left({ }^{1}\right)$. Las restricciones en el horario del sueño pueden 
conducir a privación parcial o total del sueño. La privación del sueño total se presenta si una persona no duerme, y sucede en situaciones agudas. La privación del sueño parcial está referida al sueño nocturno reducido o interrumpido, como es el caso del horario de trabajo de los médicos $\left({ }^{2}\right)$.

La pérdida del sueño es acumulativa, conduciendo al déficit de sueño ${ }^{1}{ }^{1}$. El efecto más obvio del déficit de sueño resulta en la tendencia a dormirse, lo cual se denomina somnolencia, y cuando esto sucede en circunstancias en las que la persona debería estar despierta, se denomina excesiva somnolencia diurna (ESD) $\left({ }^{3}\right)$.

Los principales factores que causan somnolencia son el déficit de sueño, pobre calidad del sueño (CS), disrupción del ritmo circadiano y uso de algunos medicamentos $\left.{ }^{1}\right)$. Una buena calidad del sueño esta referida no solo al hecho de dormir bien durante la noche, sino también a un buen funcionamiento durante la vigilia $\left({ }^{4}\right)$.

El presente estudio pretende determinar y evaluar el grado de ESD y CS en estudiantes del sexto y sétimo años de la carrera de medicina de la Universidad Peruana Cayetano Heredia (UPCH).

\section{MATERIALES Y MÉTODOS}

El estudio se realizó en una muestra no probabilística de estudiantes de medicina de la Facultad de Medicina Alberto Hurtado de la UPCH, que cursaban el sexto (externado) y sétimo (internado) año de estudios durante el año académico 2005. Los internos realizan prácticas clínicas en las sedes hospitalarias asignadas por la universidad, así como guardias, según rotación y sede docente. Los externos hacen prácticas clínicas, al igual que los internos, pero sin guardias nocturnas. El único criterio de exclusión fue diagnóstico de trastorno psiquiátrico hecho por un médi- co y reconocido explícitamente por el encuestado. El estudio fue transversal. Se aplicó tres cuestionarios. El primero contenía preguntas relacionadas a edad, sexo, actividad laboral o académica extrahospitalaria, trastorno psiquiátrico diagnosticado y consumo de tabaco y cafeína en el último mes. El segundo cuestionario fue el índice de calidad del sueño de Pittsburgh (Pittsburgh sleep quality index - PSQI) $\left({ }^{5}\right)$, versión española $\left(^{6}\right)$, que proporciona una puntuación global de la CS y puntuaciones parciales en 7 distintos componentes. La puntuación global tiene un rango de 0 a 21 y un puntaje mayor a 5 indica que el encuestado tiene mala calidad del sueño, por lo que es calificado como 'mal durmiente' $\left({ }^{6}\right)$. En población española $\left({ }^{6}\right)$ su sensibilidad es $88,6 \%$ y especificidad $74,2 \%$, y en población americana $\left(^{5}\right)$ $89,6 \%$ y $86,5 \%$, respectivamente. Medimos la homogeneidad interna de esta herramienta empleando la prueba alfa de Cronbach, encontrando alta confiabilidad $(>0,50)$.

El tercer cuestionario fue la escala de somnolencia de Epworth (Epworth sleepiness scale - ESS) $\left(^{7}\right)$, que indaga por la tendencia de quedarse dormido en 8 distintas situaciones de la vida diaria $\left({ }^{8-11}\right)$. La puntuación global tiene un rango de 0 a 24 y un puntaje mayor a 10 es considerado como positivo para la característica de ESD $\left({ }^{12}\right)$.

Los cuestionarios fueron aplicados a toda la población objetivo, en un período de 7 días. La participación fue voluntaria y anónima. Con la participación y llenado de los cuestionarios los estudiantes autorizaron su inclusión en el estudio. El estudio fue previamente revisado y aprobado por el Comité Institucional de Ética de la UPCH, con código 51042. Se registró todos los datos en una tabla del programa Excel $^{\circledR}$ v.11 y sometidos a análisis estadístico con el programa Epi-Info ${ }^{\circledR}$ v.3.3.2. Se consideró significancia estadística $p<0,05$. 


\section{RESULTADOS}

La población total de externos registrada en la base de datos de la UPCH fue 105 y participaron en el estudio 76 (72\%); se excluyó $8(11 \%)$ por trastornos psiquiátri$\cos$ y 3 por no completar correctamente los cuestionarios, quedando finalmente 65 $(62 \%)$. La población total de internos fue 103, participaron 74 (72\%); se excluyó 9 (12\%) por trastornos psiquiátricos y 3 por no completar correctamente el cuestionario; quedando finalmente $62(60 \%)$. En la Tabla 1 se muestra las características de ambas poblaciones.

El análisis estadístico no mostró diferencia en los puntajes obtenidos en ambos cuestionarios ni en los componentes del PSQI; por lo que se consideró a externos e internos como una sola población (Figura 1). La mediana de la puntuación global de la ESS fue 9 [3-22]. En cuanto al PSQI, la mediana fue 6 [0-13]. Si consideramos el punto de corte en la ESS mayor a $10 \mathrm{y}$ en el PSQI mayor a 5, encontramos que $58 \%$ de los estudiantes fueron 'malos durmientes' y $34 \%$ tuvo ESD.

La media de latencia subjetiva del sueño fue $12,9 \pm 13,9$ [0-90] minutos. El $15 \%$ de encuestados demoró en conciliar el sueño entre 30 y 60 minutos y $1 \%$ más de una hora (Figura 2). No hubo diferencia en la cantidad de horas de sueño entre externos e internos, siendo la media del total 6,0 01,1 [3-9] horas. El $65 \%$ durmió 6 horas o menos y $28 \%$ cinco horas o menos. La calidad subjetiva del sueño fue 'muy mala' y 'bastante mala' en $29 \%$; y un $30 \%$ tuvo eficiencia subjetiva del sueño menor a $85 \%$ (porcentaje del tiempo en horas dormido/horas en cama) (Figura 2). Ocho encuestados $(6,3 \%)$ aceptaron haber empleado medicación hipnótica por lo menos una vez por semana. En la Tabla 2 se muestra la distribución de los encuestados por alteraciones del sueño, según el PSQI.

En la Tabla 3 se muestra la relación del consumo de cafeína y de cigarrillos, latencia subjetiva del sueño, horas de sueño, eficiencia del sueño y empleo de medicación hipnótica, con respecto al puntaje de la ESS y PSQI.

En el análisis de correlaciones; el componente 1 del PSQI (calidad subjetiva del sueño) y puntaje total del PSQI tuvo un $\mathrm{R}=0,644(p<0,001)$. Este mismo componente y la ESS, un $\mathrm{R}=0,386(p<0,001)$. El PSQI con la ESS, un $\mathrm{R}=0,426(p<0,001)$ (Figura 3). La latencia subjetiva del sueño con el puntaje global del PSQI tuvo un $\mathrm{R}=0,227(p=0,010)$ y con la del ESS un $\mathrm{R}=0,023(p=0,797)$. La cantidad de horas de sueño con el puntaje global del PSQI,

Tabla 1. Población de externos e internos de la Universidad Peruana Cayetano Heredia.

\begin{tabular}{lccc}
\hline & Externos $(\mathrm{n}=65)$ & Internos $(\mathrm{n}=62)$ & \\
\hline Edad & $23,7 \pm 1,3[22-28]$ & $24,8 \pm 1,3[22-29]$ & $\mathrm{p}^{\circ}<0,001^{*}$ \\
Sexo: femenino/masculino & $31(48 \%) / 34(52 \%)$ & $24(39 \%) / 38(61 \%)$ & $\mathrm{NS}^{+}$ \\
Actividad extrahospitalaria & $6(9,2 \%)$ & $5(8,1 \%)$ & $\mathrm{NS}^{\dagger}$ \\
Consumo > 10 cigarros/día & $2(3,1 \%)$ & $4(6,5 \%)$ & $\mathrm{NS}^{\dagger}$ \\
Consumo diario de cafeína & $26(40,0 \%)$ & $23(37,1 \%)$ & $\mathrm{NS}^{\dagger}$ \\
Uso de medicación hipnótica & $4(6,2 \%)$ & $4(6,5 \%)$ & $\mathrm{NS}^{\dagger}$ \\
\hline
\end{tabular}

Valores se presentan: Media \pm DS [Mín-Máx] y n (\%)

NS = Estadísticamente no significativo.

* Se utilizó la prueba U-Mann Whitney con dos grados de libertad.

+ Se utilizó la prueba exacta de Fisher con dos grados de libertad. 


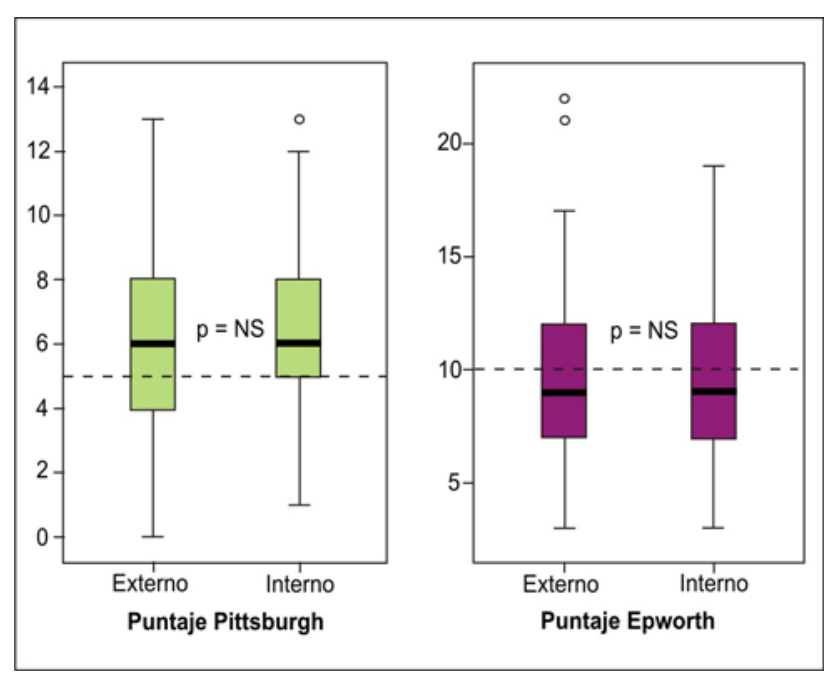

Figura 1. Puntajes del índice de calidad del sueño Pittsburgh y escala de somnolencia de Epworth en externos e internos.

un $\mathrm{R}=-0,648(p<0,001)$, y con la del ESS, un $\mathrm{R}=-0,263(p=0,003)$.

\section{DISCUSIÓN}

Horarios de trabajo variables, largas horas de jornada y períodos de guardia son muy comunes en la práctica cotidiana de médicos, tanto durante su formación como a lo largo de su actividad profesional. Estas exigencias laborales conducen a pérdida del sueño, disrupción del ritmo circadiano y fatiga del personal médico $\left({ }^{13}\right)$. Los estudiantes de medicina también están inmersos en este contexto.

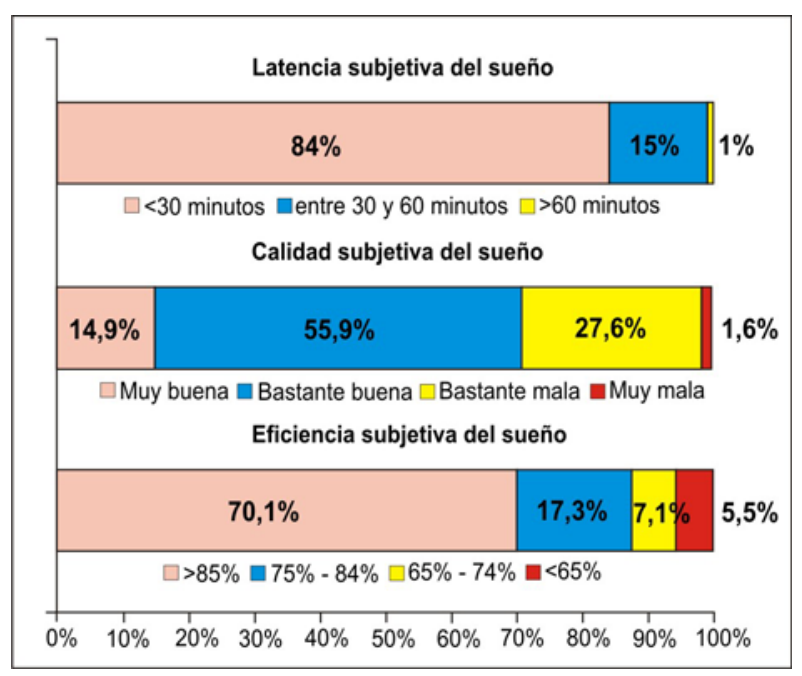

Figura 2. Distribución de encuestados según latencia, calidad y eficiencia subjetiva del sueño.

Se ha documentado entre el personal médico que la privación del sueño causa déficit en la atención, concentración, reacción y/o memoria, que podrían poner en riesgo a los pacientes $\left({ }^{14,15}\right)$. La pérdida del sueño y las guardias nocturnas tienen consecuencias en el área de la salud mental $\left({ }^{16,17}\right)$. Se ha descrito cambios en el humor, depresión, incremento del estrés y aumento del consumo de alcohol. Asimismo, están documentadas repercusiones en la vida familiar y social, así como efectos negativos en las relaciones personales y disminución del tiempo que se pasa con la familia. Por otro lado, existe información sobre el impacto negativo en la salud física, como quejas

Tabla 2. Alteraciones del sueño según índice de calidad del sueño de Pittsburgh.

\begin{tabular}{lcccc}
\hline & $\begin{array}{c}\text { Ninguna vez } \\
\text { en el mes }\end{array}$ & $\begin{array}{c}\text { Menos de una vez } \\
\text { a la semana }\end{array}$ & $\begin{array}{c}\text { Una o dos veces } \\
\text { a la semana }\end{array}$ & $\begin{array}{c}\text { Tres o más veces } \\
\text { a la semana }\end{array}$ \\
\hline No conciliar el sueño en la primera media hora & $73(57 \%)$ & $40(32 \%)$ & $8(6,3 \%)$ & $6(4,7 \%)$ \\
Despertarse durante la noche & $66(52 \%)$ & $43(34 \%)$ & $12(9,3 \%)$ & $6(4,7 \%)$ \\
No poder respirar bien & $117(92 \%)$ & $8(6,4 \%)$ & $1(0,8 \%)$ & $1(0,8 \%)$ \\
Toser o roncar ruidosamente & $113(89 \%)$ & $9(7,1 \%)$ & $3(2,3 \%)$ & $2(1,6 \%)$ \\
\hline
\end{tabular}

Valores se presentan: $n(\%)$ 
Edmundo Rosales y col.

Tabla 3. Relación de algunas variables con puntajes del índice de calidad del sueño Pittsburgh y escala de somnolencia de Epworth.

\begin{tabular}{|c|c|c|c|c|c|}
\hline & & PSQI & $p^{*}$ & ESS & $p^{*}$ \\
\hline Consumo $>10$ cigarrillos/día & $\begin{array}{c}\text { No }(n=121) \\
\text { Si }(n=6)\end{array}$ & $\begin{array}{c}6,0[4-8] \\
9,5[6-10,3]\end{array}$ & $\mathrm{p}=0,021$ & $\begin{array}{c}9,0[7-12] \\
13,5[10,8-15,3]\end{array}$ & $\mathrm{p}=0,029$ \\
\hline Consumo diario cafeína & $\begin{array}{l}\text { No }(n=78) \\
\text { Si }(n=49)\end{array}$ & $\begin{array}{c}6,0[4-7,3] \\
7,0[5-9]\end{array}$ & $\mathrm{p}=0,027$ & $\begin{array}{c}8,5[6,8-11] \\
10,0[7-14]\end{array}$ & $\mathrm{p}=0,032$ \\
\hline Latencia subjetiva del sueño $\geq 30$ min & $\begin{array}{c}\text { No }(n=107) \\
\operatorname{Si}(n=20)\end{array}$ & $\begin{array}{c}6,0[4-7] \\
8,5[6-10]\end{array}$ & $\mathrm{p}=0,001$ & $\begin{array}{c}9,0[7-12] \\
10,0[8-14]\end{array}$ & NS \\
\hline Dormir $\leq 6$ horas & $\begin{array}{l}\text { No }(n=45) \\
\text { Si }(n=82)\end{array}$ & $\begin{array}{l}4,0[3-6] \\
7,0[5-9]\end{array}$ & $\mathrm{p}<0,001$ & $\begin{array}{c}8,0[6-10,5] \\
9,0[7-13]\end{array}$ & NS \\
\hline Dormir $\leq 5$ horas & $\begin{array}{c}\text { No }(n=92) \\
\text { Si }(n=35)\end{array}$ & $\begin{array}{c}5,0[4-7] \\
8,0[6-10]\end{array}$ & $\mathrm{p}<0,001$ & $\begin{array}{c}8,0[7-11] \\
11,0[8-14]\end{array}$ & $\mathrm{p}=0,004$ \\
\hline Eficiencia del sueño $<85 \%$ & $\begin{array}{c}\text { No }(n=89) \\
\operatorname{Si}(n=38)\end{array}$ & $\begin{array}{c}5,0[4-6] \\
8,5[8-10]\end{array}$ & $\mathrm{p}<0,001$ & $\begin{array}{c}9,0[7-12] \\
10,0[7-14]\end{array}$ & NS \\
\hline Uso medicación hipnótica & $\begin{array}{l}\text { No }(n=119) \\
\operatorname{Si~}(n=8)\end{array}$ & $\begin{array}{c}6,0[4-8] \\
9,0[7-11]\end{array}$ & $\mathrm{p}=0,003$ & $\begin{array}{c}9,0[7-12] \\
10,0[8,3-12]\end{array}$ & NS \\
\hline
\end{tabular}

Valores se presentan: Mediana [percentil 25 - percentil 75].

$N S=$ Estadísticamente no significativo

* Se utilizó la prueba U-Mann Whitney con dos grados de libertad.

somáticas, incremento de complicaciones en el embarazo, incremento en el riesgo de accidentes vehiculares e impedimentos para el aprendizaje $\left({ }^{13,18-20}\right)$. Finalmente, se ha observado que luego de una privación del sueño de 24 horas, el rendimiento psicomotor disminuye en las primeras horas de la mañana, de manera similar o peor al producido por una intoxicación alcohólica con niveles de concentración en sangre mayor de $0,10 \%\left({ }^{21}\right)$.

Actualmente, existe gran controversia en la comunidad médica respecto a la magnitud y significado de la disminución del rendimiento causado por privación del sueño. Las diversas opiniones sobre la reducción de horarios de trabajo están influenciadas por el impacto de éste sobre la economía, pues habría que contratar más personal para cubrir horarios y de esa manera garantizar la continuidad y calidad del cuidado de los pacientes $\left({ }^{22-24}\right)$. Hasta donde tenemos conocimiento, en el Perú no hay estudios realizados en esta área.
En el presente estudio, los encuestados tenían labores hospitalarias similares, diferenciándose únicamente en el hecho que los internos realizaban guardias nocturnas. Por esa razón, nuestra hipótesis inicial fue que

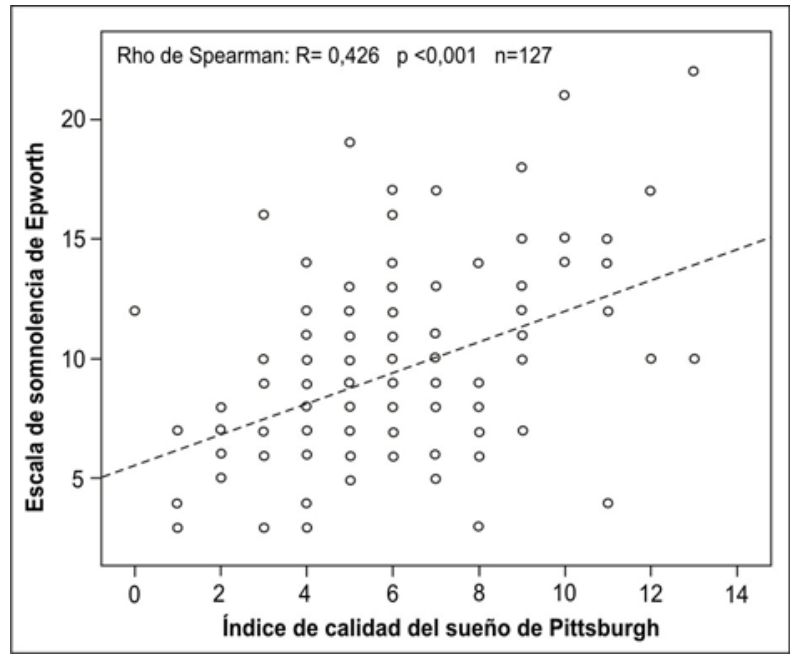

Figura 3. Correlación puntaje índice de calidad del sueño de Pittsburg y escala somnolencia de Epworth. 
existía diferencia entre las poblaciones. No obtuvimos diferencias estadísticamente significativas en los puntajes de ambas escalas. No hemos encontrado referencias en la literatura donde se compare los parámetros estudiados en poblaciones semejantes a las de nuestro estudio. Estos resultados podrían sugerir que hacer guardias nocturnas no afecta la CS ni la ESD en los estudiantes de medicina, pero debido a que nuestro estudio tiene diseño transversal no puede establecerse esta relación causal.

Más de la mitad dormía seis horas o menos, lo que conlleva a una privación crónica del sueño $\left(^{1,2}\right)$. Análogamente, más de la mitad fueron 'malos durmientes'. Sierra y col. $\left({ }^{4}\right)$ encontraron en estudiantes universitarios españoles un resultado similar al nuestro, de 60\%; en cambio, Báez y col. $\left.{ }^{25}\right)$ describieron un porcentaje mayor, que llegó a $82 \%$ entre estudiantes de medicina de una universidad colombiana. Estudios epidemiológicos realizados en población general adulta indican una prevalencia de mala calidad del sueño entre 15 a $35 \%\left({ }^{5}\right)$.

Los estudiantes universitarios están sometidos a carga curricular muy demandante y en el caso específico de los estudiantes de medicina se agrega el estrés de estar en contacto con el sufrimiento humano y la muerte. Los resultados obtenidos en la literatura son muy variables, lo que podría sugerir que la demanda curricular, hábitos de sueño y horarios de trabajo varían en cada región. De otro lado, las poblaciones en cuestión no son similares y las herramientas empleadas no son exactamente las mismas. En nuestro estudio, los estudiantes de medicina dormían 6,03 horas en promedio. En cambio, Lima y col. $\left({ }^{26}\right)$, en un estudio en estudiantes de medicina en Brasil, encontró que estos dormían 6,62-7,28 horas; lo que hace una diferencia de media a una hora. Esta información podría explicar la razón por la que Lima informó un menor porcentaje de 'malos durmientes'. Ello concuerda con nuestro hallazgo de una asocia- ción negativa entre cantidad de horas de sueño y el puntaje PSQI, y que el dormir 6 horas o menos está relacionado con mayores puntajes en dicha escala. También, encontramos asociación negativa entre la cantidad de horas de sueño con la ESS, la que no fuera encontrada por Aloé y col en estudiantes de medicina de Brasil $\left({ }^{27}\right)$.

En cuanto a la ESD, se observó que la tercera parte de la muestra tenía puntaje mayor a 10 en la ESS. Moo-Estrella y col. describen una cifra similar de $31 \%$ en estudiantes universitarios brasileños $\left({ }^{28}\right)$. Esta variable fue distinta al estudio de Rodrigues y col. $\left({ }^{29}\right)$, también realizada en estudiantes de medicina brasileños, con $39 \%$ de ESD al inicio del semestre y $62 \%$ al finalizarlo. Los autores mencionan que los estudiantes con cansancio y somnolencia no tuvieron un desempeño académico adecuado. Frecuentemente, cursaban con mal humor y a veces llegaban a deprimirse, lo que agravaba su somnolencia. Cuando se evaluó esta característica en población general, Souza y col. $\left({ }^{30}\right)$, en un estudio comunitario realizado en Brasil usando la ESS, encontró $18,9 \%$ de ESD.

La correlación entre puntajes globales de ambas escalas fue positiva. Similar hallazgo encontraron Souza y col. $\left({ }^{31}\right)$, en un estudio realizado en choferes brasileños de camión. Aunque el tipo de diseño de ambos estudios no determina la dirección de la correlación, es lógico asumir que en las personas con mala CS la somnolencia se incrementa y no a la inversa.

Las proporciones encontradas de latencia subjetiva del sueño prolongada $(>=30 \mathrm{mi}-$ nutos) fueron similares a las informadas por Baéz y col. $\left({ }^{25}\right)$, quienes la encontraron en $15 \%$ de estudiantes de medicina colombianos. En el estudio de Sierra y col. $\left({ }^{4}\right)$ llegó a $50 \%$. El grupo de estudiantes colombianos $\left({ }^{25}\right)$ obtuvo resultados similares al nuestro, con relación a la latencia del sueño; pero, los autores informaron mayor porcen- 
taje de 'malos durmientes'. En cambio, en la publicación de Sierra y col., en estudiantes españoles $\left({ }^{4}\right)$, se encontró mayor porcentaje de estudiantes con latencia del sueño mayor a 30 minutos, pero con porcentaje similar al nuestro en lo que a 'malos durmientes' se refiere.

Aunque el objetivo del estudio y las herramientas empleadas no sirven para establecer diagnósticos de desórdenes del sueño, consideramos pertinente hacer algunos comentarios. El $42,5 \%$ presentó dificultades para conciliar el sueño en la primera media hora y $48 \%$ tuvo despertares nocturnos. Estos resultados indicarían insomnio de conciliación o de mantenimiento en proporciones elevadas ${ }^{32}$ ). De otro lado, $11 \%$ roncaba ruidosamente, lo que configura el principal síntoma del síndrome de apneashipopneas del sueño (SAHS) $\left.{ }^{(33}\right)$. Los síntomas asociados a trastornos del sueño son frecuentes en nuestra población $\left(^{34-37}\right)$. Cerca de $30 \%$ a $40 \%$ de adultos presentan algún nivel de insomnio y aproximadamente en $10 \%$ a $15 \%$, el insomnio es crónico y/o severo $\left.{ }^{32}\right)$. En cuanto al SAHS, éste es un desorden común y su prevalencia en estudios foráneos puede fluctuar entre 2 y $4 \%$ de los adultos en la edad media de la vida ${ }^{(38,39}$ ).

El componente 1 del PSQI indicó que más de dos tercios de los estudiantes califica su calidad del sueño como 'bastante buena' o 'buena', resultado muy similar al de Báez y col., de $73 \%\left({ }^{25}\right)$, y Sierra y col., 69\% ( $\left.{ }^{4}\right)$. Es decir, 29\% califica su calidad del sueño como 'mala' o 'bastante mala'. Ello difiere del puntaje global del PSQI, donde 58\% fue calificado como 'mal durmiente'. Estos resultados aparentemente discrepantes entre calificación subjetiva del encuestado y el puntaje global del cuestionario reflejarían una sobreestimación de la calidad subjetiva del sueño por parte de los encuestados; algo similar a lo observado cuando se evalúa subjetivamente la somnolencia $\left({ }^{40,41}\right)$.
El porcentaje de encuestados que empleaba medicación hipnótica en nuestro estudio fue $6,3 \%$. En estudiantes de medicina colombianos fue $9,5 \%\left({ }^{25}\right)$ y estudiantes universitarios españoles $13 \%\left({ }^{4}\right)$. Nosotros encontramos que el uso de estos medicamentos estuvo relacionado con peor calidad del sueño. Un estudio realizado en Perú describe que las benzodiazepinas son los medicamentos hipnóticos más usados $\left({ }^{36}\right)$.

En dos estudios hechos en estudiantes universitarios, se intentó ver el efecto de algunas sustancias sobre la calidad del sueño. Báez y col. $\left({ }^{25}\right)$ encontraron que el uso de sustancias estimulantes, como cafeína, nicotina, té o mate, ejercían un efecto negativo en la calidad del sueño. Asimismo, Sierra y col. $\left({ }^{4}\right)$ describieron que la cafeína, alcohol y tabaco presentaban el mismo efecto, aunque en ambos estudios no se analizó las sustancias por separado. En nuestro estudio, los encuestados que consumían tabaco o cafeína tuvieron mayor puntaje en el PSQI y ESS.

Nuestro estudio tiene algunas limitaciones. Los resultados obtenidos de los cuestionarios se basan en un autorreporte sustentado en la apreciación subjetiva del encuestado. Esta es una limitación conocida de los cuestionarios. La somnolencia y la calidad del sueño son variables subjetivas y están sujetas a variabilidad individual, pudiendo llegar incluso a confundir la somnolencia con el cansancio $\left({ }^{42}\right)$. El estudio también tiene limitaciones inherentes a la recopilación de información por medio de cuestionarios, como el sesgo de memoria y consideraciones subjetivas que podrían influenciar en las respuestas sobre el número de horas de sueño o la cantidad de minutos transcurridos hasta conciliar el sueño. Algunas personas con privación crónica del sueño subestiman sus síntomas $\left({ }^{40,41}\right)$ hecho que podría darse en la población encuestada. No consignamos en el cuestionario información específica sobre la cantidad de guardias y/o siestas diurnas realizadas durante 
el mes. Finalmente, la relación causal de algunas variables sobre la CS o ESD no puede ser establecida, ya que el diseño fue transversal. A pesar de ello, consideramos que tanto la escala de somnolencia Epworth como el cuestionario de Pittsburg son herramientas sencillas, económicas y útiles para calificar somnolencia y calidad de sueño en población peruana.

En conclusión, no existe diferencia en el grado de CS y ESD entre externos e internos de medicina de la UPCH. Registramos alta frecuencia de pobre CS y ESD entre los encuestados. Dichos parámetros se agravan con el consumo de tabaco y cafeína, latencia subjetiva del sueño prolongada, corta duración del sueño, eficiencia del sueño menor de $85 \%$ y uso de medicación hipnótica. Finalmente, encontramos una correlación entre la CS y ESD.

Es necesario implementar estudios para cuantificar los efectos de la CS y la ESD en el rendimiento académico y profesional de estudiantes de medicina del Perú. Los estudios deberían extenderse a médicos residentes y médicos asistentes. Esta información permitirá implementar medidas específicas relacionadas a una adecuada programación de horarios de trabajo, guardias y periodos de descanso, que promuevan buena higiene del sueño en profesionales de la salud y que garantice excelencia en la atención de los pacientes.

\section{REFERENCIAS BIBLIOGRÁFICAS}

1. Howard SK. Sleep deprivation and fatigue. En: Miller RD. Anesthesia. 5th edition. Philadelphia: Churchill Livingstone; 2000:2637-46.

2. Weinger MB, Ancoli-Israel S. Sleep deprivation and clinical performance. JAMA. 2002;287:955-7.

3. Cluydts R, De Valck E, Verstraeten E, Theys P. Daytime sleepiness and its evaluation. Sleep Med Rev. 2002;6:8396.

4. Sierra JC, Jiménez-Navarro C, Martín-Ortíz JD. Calidad del sueño en estudiantes universitarios: importancia de la higiene del sueño. Salud Mental. 2002;25:35-43.
5. Buysse DJ, Reynolds CF, Monk TH, Berman SR, Kupfer DJ. The Pittsburgh Sleep Quality Index: A new instrument for psychiatric practice and research. Psychiatry Res. 1989;28:193-213.

6. Royuela A, Macías JA. Propiedades clinimétricas de la versión castellana del Cuestionario de Pittsburgh. VigiliaSueño. 1997;9:81-94.

7. Johns MW. A new method for measuring daytime sleepiness: The Epworth Sleepiness Scale. Sleep. 1991;14:540-5.

8. Ferrer M, Vilagut G, Monasterio C, Montserrat JM, Mayos M, Alonso J. Medida del impacto de los trastornos del sueño: las versiones españolas del cuestionario del impacto funcional del sueño y de la Escala de Somnolencia de Epworth. Med Clin (Barc). 1999;113:250-5.

9. Chiner E, Arriero JM, Signes-Costa J, Marco J, Fuentes I. Validation of the spanish version of the Epworth Sleepiness Scale in patients with a sleep apnea syndrome. Arch Bronconeumol. 1999;35:422-7.

10. Rey de Castro J, Vizcarra D, Álvarez J. Somnolencia diurna y síndrome apnea hipopnea del sueño - Asociación entre parámetros antropométricos y puntaje Epworth en polisomnografía convencional y de noche partida. Rev Soc Peru Med Interna. 2003;16:74-83.

11. Rey de Castro J, Vizcarra D. Limited value of Epworth Modified Scale for hipersomnia assessment in 57 patients submited to polisomnogrphy. Libro de Resúmenes $24^{\text {th }}$ International Congress of Internal Medicine. PS 134. 1998. p. 80.

12. Johns M, Hocking B. Daytime sleepiness and sleep habits of Australian workers. Sleep. 1997;20:844-9.

13. Howard SK, Gaba DM, Rosekind MR, Zarcone VP. The risks and implications of excessive daytime sleepiness in resident physicians. Acad Med. 2002;77:1019-25.

14. Green MJ. What (if anything) is wrong with residency overwork? Ann Intern Med. 1995;123:512-7.

15. Arnedt JT, Owens J, Crouch M, Stahl J, Carskadon MA. Neurobehavioral performance of residents after heavy night call vs after alcohol ingestion. JAMA. 2005;294:1025-33.

16. Bellini LM, Shea JA. Mood change and empathy decline persist during three years of internal medicine training. Acad Med. 2005;80:164-7.

17. Smith-Coggins R, Rosekind MR, Hurd S, Buccino KR. Relationship of day versus night sleep to physician performance and mood. Ann Emerg Med. 1994;24:959-61.

18. Horne J, Reyner L. Vehicle accidents related to sleep: a review. Occup Environ Med. 1999;56:289-94.

19. Barger LK, Cade BE, Ayas NT, Cronin JW, Rosner B, Speizer FE, et al. Extended work shifts and the risk of motor vehicle crashes among interns. N Eng J Med. 2005;352:125-34.

20. Owens JA. Sleep loss and fatigue in medical training. Curr Opin Pulm Med. 2001;7:411-8.

21. Dawson D, Reid K. Fatigue, alcohol and performance impairment. Nature. 1997;388:235.

22. Steinbrook R. The debate over residents' work hours. N Eng J Med. 2002;347:1296-302. 
23. Gaba DM, Howard SK. Patient safety: fatigue among clinicians and the safety of patients. $\mathrm{N}$ Eng $\mathrm{J}$ Med. 2002;347:1249-55

24. Volpp KGM, Grande D. Residents' suggestions for reducing errors in teaching hospitals. N Eng J Med. 2003;348:851-5.

25. Báez GF, Flores NN, González TP, Horrisberger HS. Calidad del sueño en estudiantes de medicina. Revista de Posgrado de la VIa Cátedra de Medicina. 2005;141:14-7.

26. Lima PF, Medeiros ALD, Araujo JF. Sleep-wake pattern of medical students: early versus late class starting time. Braz J Med Biol Res. 2002;35:1373-7.

27. Aloé F, André T, Stella M. Epworth sleepiness scale outcome in 616 Brazilian medical students. Arq Neuropsiquiatr. 1997;55:220-6.

28. Moo-Estrella J, Pérez-Benítez H, Solís-Rodríguez F, Arankowsky-Sandoval G. Evaluation of depressive symptoms and sleep alterations in college students. Arch Med Res. 2005;36:393-8.

29. Rodrigues RND, Viegas CAA, Abreu e Silva AAA, Tavares $P$. Daytime sleepiness and academic performance in medical students. Arq Neuropsiquiatr. 2002;60:6-11.

30. Souza JC, Magna LA, Reimao R. Excessive daytime sleepiness in Campo Grande general population, Brazil. Arq Neuropsiquiatr. 2002;60:558-62.

31. Souza JC, Paiva T, Reimão R. Sleep habits, sleepiness and accidents among truck drivers. Arq Neuropsiquiatr. 2005;63:925-30.

32. National Center on Sleep Disorders Research and Office of Prevention, Education, and Control. Insomnia: Assessment and Management in Primary Care. National Heart, Lung, and Blood Institute, National Institutes of Health. Bethesda: NIH Publication. No. 98-4088. Septiembre 1998. p. 16

33. Rey de Castro J, Vizcarra D. Síndrome apnea sueño obstructiva. Descripción clínica y polisomnográfica en 48 pacientes. Rev Soc Peru Med Interna. 1999;12:2-7.

34. Rey de Castro J, Álvarez J, Gaffo A. Síntomas relacionados a trastornos del sueño en supuestos sanos que asisten a un centro de Atención Primaria de Salud. Rev Med Hered. 2005; 16:31-8.

35. Rey de Castro J, Vizcarra D. Frecuencia de síntomas del síndrome de apnea hipopnea del sueño e insomnio en médicos de una clínica privada peruana. Rev Med Hered. 2003;14:53-8.
36. Pacheco GM, Rey de Castro J. Insomnio en pacientes adultos ambulatorios de medicina interna del Hospital Nacional Arzobispo Loayza. Rev Med Hered. 2003;14:63-8.

37. Rey de Castro J, Hernández-Montalvo J. Síntomas relacionados al síndrome de apnea-hipopnea obstructivo del sueño e insomnio ¿Son frecuentes en el consultorio de neumología del adulto?. Rev Soc Peru Med Interna. 2000;13:196-200.

38. Young T, Palta M, Dempsey J, Skatrud J, Weber S, Badr S. The occurrence of sleep-disordered breathing among middle-aged adults. N Eng J Med. 1993;328:1230-5.

39. Duran J, Esnaola S, Rubio R, Iztueta AA. Obstructive sleep apnea-hipopnea and related clinical features in a populationbased sample of subjects aged 30 to 70 years. Am J Respir Crit Care Med. 2001;163:685-9.

40. Wiggins CL, Schmidt-Nowara WW, Coultas DB, Samet JM. Comparison of self- and spouse reports of snoring and other symptoms associated with sleep apnea syndrome. Sleep. 1990;13:245-52.

41. García P, Capote F, Quintana ME, Fuentes MA, Carmona C, Sánchez A. Valoración mediante escala de Epworth de la somnolencia diurna en pacientes con sospecha de síndrome de apneas obstructivas durante el sueño. Diferencias entre los pacientes y sus parejas. Arch Bronconeumol. 2000;36:608-11.

42. Rey de Castro J, Gallo J, Loureiro H. Cansancio y somnolencia en conductores de ómnibus y accidentes de tránsito en el Perú: estudio cuantitativo. Rev Panan Salud Pública. 2004;16:11-8.

Manuscrito recibido el 06 de junio de 2007 y aceptado para publicación el 20 de setiembre de 2007.

Correspondencia:

Edmundo Rosales Mayor

Fidel Olivas Escudero 151 Dpto 301, San Miguel

Lima 32, Perú

Correo-e:08251@upch.edu.pe 